\title{
Molecular gas dynamics in the Rosette Nebula
}

\author{
Henry E. Matthews ${ }^{1}$ and William R. F. Dent ${ }^{2}$ \\ ${ }^{1}$ Herzberg Institute of Astrophysics, National Research Council of Canada, Victoria, BC \\ V2E 2E7, Canada \\ email: henry.matthews@nrc-cnrc.gc.ca \\ ${ }^{2}$ Royal Observatory Edinburgh, Scotland, UK \\ email: :dent@roe.ac.uk
}

\begin{abstract}
We present observations of the Rosette Nebula and its near environment in the CO 3-2 transition obtained with an angular resolution of $20^{\prime \prime}$. The gas dynamics of the region are complex; we find (1) a ring of gas expanding at about $20 \mathrm{~km} \mathrm{~s}^{-1},(2)$ a number of collimated outflow sources, and (3) a chain of dust clumps having a velocity gradient along its length.
\end{abstract}

Keywords. ISM: molecules, ISM: kinematics and dynamics, instrumentation: detectors

\section{Observations and Data Reduction}

The Rosette Nebula, at a distance of 1400 pc, is a textbook example of the interaction between OB stars and surrounding gas and dust. NGC 2244, the central star cluster, contains six O-type stars with a total luminosity of $1000 \mathrm{~L}_{\odot}$.

The data were obtained in 2006 and 2007 using a completely new suite of instrumentation at the James Clerk Maxwell Telescope (JCMT), on Mauna Kea, Hawaii, following major upgrades to the telescope and its observing capabilities. HARP, a 16-receptor (square $4 \times 4$ ) array receiver (Smith et al. 2003$)$ operating in the $325-375 \mathrm{GHz}(850 \mu \mathrm{m})$ region, was used with ACSIS, the spectral line correlator backend (Hovey et al. 2000) and a new telescope control system (OCS; Rees et al. 2002). The observations were carried out by continuously recording the data while the telescope was scanned across the target field. The scan rate used was $75^{\prime \prime}$ per second, with a sample rate of $10 \mathrm{~Hz}$. The entire region was scanned in both RA and Declination directions, ultimately resulting in a basket-weaved image solution with a well-determined and flat baselevel. The raw spectral resolution of these data was $0.42 \mathrm{~km} \mathrm{~s}^{-1}$.

Data processing employed the SMURF package (Jenness et al. 2008) to first convert the data to spectral cubes. The Starlink KAPPA and CCDPACK routines were then used to remove a linear baseline, transform to a regular grid and combine the data into a single cube. Of these data, only 66 spectral channels were retained, covering the velocity interval of -3 through $25 \mathrm{~km} \mathrm{~s}^{-1}$ appropriate for the Rosette Nebula. Subsequent smoothing of the data to an angular resolution of $20^{\prime \prime}$ resulted in a data cube in which the rms noise per point was $0.3 \mathrm{~K} \mathrm{~T}_{A}^{*}$ (corrected antenna temperature scale).

\section{Results}

The CO 3-2 image of the Rosette Nebula (see Figure 1, and Dent et al. 2008) obtained from these data shows that molecular material extends over a substantially larger area than the optical extent of the nebula. The structural features of the $\mathrm{CO}$ emission fall into four main types: compact flows, clumps, smooth extended regions, and elongated structures with narrow line velocity widths. 
The data reveal a ring of gas expanding at $20 \mathrm{~km} \mathrm{~s}^{-1}$, and show that compact, relatively high-velocity flows tend to be associated with the known IRAS sources, of which AFGL 961 is the most well-known, correlated with young star clusters. The CO 3-2/1-0 ratios indicate excitation temperatures of about $30-60 \mathrm{~K}$ in these regions. In a galactic context, these are relatively low energy outflows, having little influence on the Rosette Molecular Cloud.

Clumps of gas, typically $15^{\prime \prime}$ to $3^{\prime}$ in size, are common. We estimate that in the present work we are able to detect such objects with masses as low as perhaps 30-100 Jupiter masses. Of particular note is a chain of clumps in the north-west quadrant of the Rosette which corresponds with a group of dust globules and elephant truck structures (Gahm et al. 2006) seen against the nebular background. The velocity information from these and other objects suggest that these objects are part of an expanding partial ring. The expansion rate of this structure, $16 \mathrm{~km} \mathrm{~s}^{-1}$, suggests a dynamical age of $0.8 \mathrm{Myr}$, as compared with a systemic age of 2-3 Myr (Balog et al. 2007).

Kinetic temperatures derived from the CO 1-0 and 3-2 integrated line intensities are in the range $25-45 \mathrm{~K}$. The clump mass distribution is estimated to be complete to about $0.1 \mathrm{M}_{\odot}$. For optically thin $\mathrm{CO}$ emission $\left(\mathrm{A}_{v} \leqslant 1\right)$ we find a mass-number slope of -0.6 (i.e., $d N(m) / d M \sim-1.6$ ) for the clump mass range $0.1-1.0 \mathrm{M}_{\odot}$.

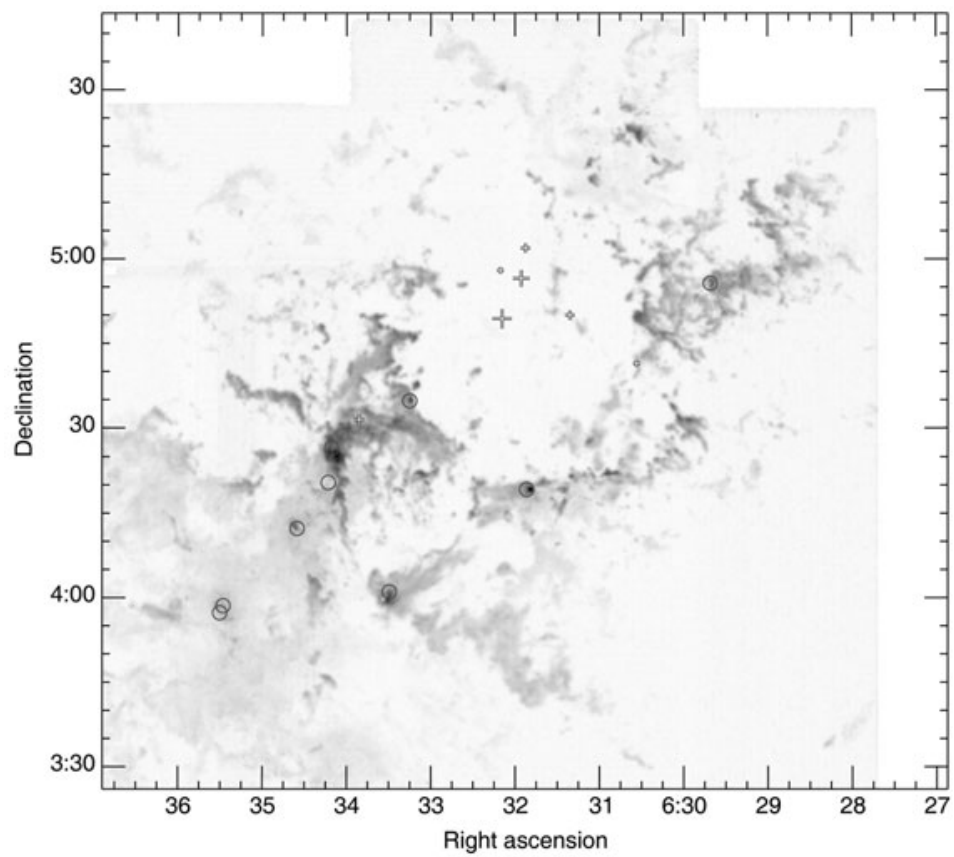

Figure 1. A molecular line image of the Rosette Nebula seen in emission from CO (3-2 transition). The image is about 2 degrees across, about twice the extent of the visible nebula. The positions of the luminous O-stars are indicated by crosses just above the centre of the image, and the circles show the locations of outflow sources.

\section{References}

Balog, Z., et al. 2007, ApJ, 660, 1532

Dent, W. R. F. D., et al. 2008, MNRAS, in preparation

Gahm, G. F., et al. 2006, A\&SA, 454, 201

Hovey, G., et al. 2000, SPIE, 4015, 114

Jenness, T., et al. 2008, ADASS XVIII, in press

Rees, N., et al. 2002, ASPC, 281, 500

Smith, H., et al. 2003, SPIE, 4855, 338 